\title{
Biomarkers in autoimmune rheumatic diseases
}

\author{
"I hope the highlighted articles presented in this themed \\ issue will encourage readers to discuss, debate and reconsider \\ the current and future prospects for biomarker research in \\ autoimmune rheumatic disorders"
}

Keywords: diagnosis • psoriatic arthritis • rheumatoid arthritis • systemic lupus erythematosus

Doctor-diagnosed arthritis, the most common group of rheumatic disorders, is estimated by the CDC to affect 52.5 million US adults, approximately one in five [1]. Arthritis is documented to be the leading cause of disability in the USA, limiting the activity of 22.7 million adults [1].

The themed articles in this issue of Biomarkers in Medicine aim to outline biomarker identification, development, validation and implementation efforts in three autoimmune rheumatic diseases: rheumatoid arthritis (RA); psoriatic arthritis (PsA) and systemic lupus erythematosus (SLE).

WHO figures estimate global RA prevalence to vary between 0.3 and $1 \%$ [2], onset is commonly between 20 and 40 years of age and the disease results in an inability to maintain a full-time job in $50 \%$ of patients within 10 years of diagnosis [2]. Figures in 2009 put the cost of RA to the UK economy alone at $£ 4.8$ billion annually [3]. Over recent years, the introduction of biologic drugs has led to significant changes in the management of RA [4]. As one of the most successful classes of biologics employed in this disorder, anti-TNF agents have come to dominate the field. However, anti-TNF agents exhibit an inadequate response in $30-40 \%$ of treated RA patients [4]. In the first review article in this issue, Oliver et al. discuss the potential for genomic and genetic markers to inform anti-TNF treatment for RA. The authors outline a range of potential markers for use in treatment monitoring, in addition to examining barriers to progress in this area, next steps for the field and alterna- tive approaches, including transcriptomics and epigenetics [4].

Unlike RA, PsA is associated with skin lesions (psoriasis); however, the two remain difficult to differentiate. Such difficulties render accurate estimates of PsA prevalence difficult to obtain with European and US estimates varying from $0.02-0.42 \%$ [5]. In their review article in this issue, Caruntu et al. discuss the current status of biomarkers in PsA [6]. The authors consider the roles of immune pathways in PsA and their potential in aiding the identification of prospective protein markers. Genomic markers are also reviewed, in addition to discussion of autoantibodies with potential to stratify PsA into risk sub groups. The authors express hope for the translation of these markers into targets for immune therapies and the subsequent need for markers to monitor such therapies this would create [6].

The last two themed reviews in this issue address biomarkers in pediatric SLE. Childhood-onset SLE is a rare condition, with a prevalence of just 3.3-8.8 per 100,000 children [7]. In the first of these articles, the authors discuss markers for identifying and monitoring kidney involvement in pediatric SLE. As 50-80\% of adolescents diagnosed with SLE develop kidney involvement, and therefore increased risk of morbidity, the need for effective biomarkers to reduce reliance on renal biopsy for identification is paramount [8]. Goliav et al. discuss current approaches to classifying lupus nephritis, review current clinical serum and urine markers, in addition to considering blood and urine markers in experimental stages, and finally cover genetic and epigenetic factors [8].

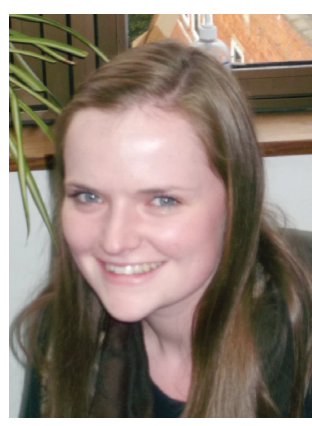

Hannah Wilson

Future Medicine Ltd, Unitec House, 2 Albert Place, Finchley Central, N3 1QB, London, UK

hannah.wilson@futuremedicine.com 
In their second paper, the group employ a similar approach to markers for CNS involvement in pediatric SLE [9]. Following discussion of disease pathogenesis and classification, the authors cover both established and experimental biomarkers, including those found in the blood and CSF, in addition to neuroimaging and cognitive testing approaches [9].

In summary, I hope the highlighted articles presented in this themed issue will encourage readers to discuss, debate and reconsider the current and future prospects for biomarker research in autoimmune rheumatic disorders.

\section{References}

1 Arthritis: meeting the challenge of living well. www.cdc.gov

2 Chronic diseases and health promotion. www.who.int/chp/topics/rheumatic/en/

3 National Audit Office. Services for people with rheumatoid arthritis.

www.nao.org.uk

4 Oliver J, Plant D, Webster AP, Barton A. Genetic and genomic markers to inform treatment of rheumatoid arthritis with anti-TNF drugs. Biomarkers Med. 9(6), 499-512 (2015).

5 Liu JT, Yeh HM, Liu SY, Chen KT. Psoriatic arthritis: epidemiology, diagnosis, and treatment. World J. Orthoped. 5(4), 537-543 (2014).

\section{Disclaimer}

$\mathrm{H}$ Wilson is an employee of Future Science Group.

\section{Financial \& competing interests disclosure}

The author has no relevant affiliations or financial involvement with any organization or entity with a financial interest in or financial conflict with the subject matter or materials discussed in the manuscript. This includes employment, consultancies, honoraria, stock ownership or options, expert testimony, grants or patents received or pending, or royalties.

No writing assistance was utilized in the production of this manuscript.

6 Caruntu C, Boda D, Dumitrascu Constantin C, Neagu M. Proteomics focusing on immune markers in psoriatic arthritis. Biomarkers Med. 9(6), 513-528 (2015).

7 Levy DM, Kamphuis S. Systemic lupus erythematosus in children and adolescents. Pediatr. Clin. North Am. 59(2), 345-364 (2012).

8 Goliav B, Putterman C, Rubinstein T. Biomarkers for kidney involvement in pediatric lupus. Biomarkers Med. 9(6), 529-543 (2015)

9 Rubinstein T, Putterman C, Goliav B. Biomarkers for central nervous system involvement in pediatric lupus. Biomarkers Med. 9(6), 545-558 (2015). 